\title{
Biomechanical demands on posterior fusion instrumentation during lordosis restoration procedures
}

\author{
Calvin C. Kuo, MD, ${ }^{1}$ Audrey Martin, BS, ${ }^{2}$ Connor Telles, MD, ${ }^{3}$ Jeremi Leasure, $M S,{ }^{2}$ Alex lezza, MD, ${ }^{4}$ \\ Christopher Ames, MD, and Dimitriy Kondrashov, MD ${ }^{6,7}$ \\ ${ }^{1}$ Kaiser Permanente Medical Center, Oakland; ${ }^{2}$ The Taylor Collaboration, San Francisco; ${ }^{3}$ Sierra Pacific Orthopedics, Fresno; \\ ${ }^{4}$ Redwood Orthopaedic Surgery Associates, Santa Rosa; ${ }^{5}$ CSF Department of Neurological Surgery, San Francisco; ${ }^{6}$ SF \\ Orthopaedic Residency Program, San Francisco; and 'St. Mary's Spine Center, San Francisco, California
}

\begin{abstract}
OBJECTIVE The goal of this study was to investigate the forces placed on posterior fusion instrumentation by 3 commonly used intraoperative techniques to restore lumbar lordosis: 1) cantilever bending; 2) in situ bending; and 3) compression and/or distraction of screws along posterior fusion rods.
\end{abstract}

METHODS Five cadaveric torsos were instrumented with pedicle screws at the L1-5 levels. Specimens underwent each of the 3 lordosis restoration procedures. The pedicle screw pullout force was monitored in real time via strain gauges that were mounted unilaterally at each level. The degree of correction was noted through fluoroscopic imaging. The peak loads experienced on the screws during surgery, total demand on instrumentation, and resting loads after corrective maneuvers were measured.

RESULTS A mean overall lordotic correction of $10.9 \pm 4.7^{\circ}$ was achieved. No statistically significant difference in lordotic correction was observed between restoration procedures. In situ bending imparted the largest loads intraoperatively with an average of $1060 \pm 599.9 \mathrm{~N}$, followed by compression/distraction $(971 \pm 534.1 \mathrm{~N})$ and cantilever bending (705 $\pm 413.0 \mathrm{~N})$. In situ bending produced the largest total demand and postoperative loads at L-1 (1879 \pm 1064.1 and $487 \pm$ $118.8 \mathrm{~N}$, respectively), which were statistically higher than cantilever bending and compression/distraction $(786 \pm 272.1$ and $138 \pm 99.2 \mathrm{~N}$, respectively).

CONCLUSIONS In situ bending resulted in the highest mechanical demand on posterior lumbar instrumentation, as well as the largest postoperative loads at L-1. These results suggest that the forces generated with in situ bending indicate a greater chance of intraoperative instrumentation failure and postoperative proximal pedicle screw pullout when compared with cantilever bending and/or compression/distraction options. The results are aimed at optimizing correction and fusion strategies in lordosis restoration cases.

http://thejns.org/doi/abs/10.3171/2016.1.SPINE15264

KEY WORDS lumbar; lordosis; intraoperative; posterior; fusion; balance; sagittal; correction; bending; cantilever

$\mathrm{R}$ ESTORATION of sagittal balance is one of the most important considerations in adult spinal reconstructive surgery. Positive sagittal balance has been identified as the radiographic parameter that is most correlated with adverse health status outcomes in spinal deformity patients. ${ }^{6}$ Restoration of optimal lumbar lordosis and secure lumbosacral fixation are necessary, especially in patients with preoperative sagittal imbalance and high pelvic incidence, in order to prevent sagittal decompensation after surgery. ${ }^{3}$ Furthermore, restoration of lower lumbar lordosis in patients with degenerative flat back syndrome has been shown clinically to result in additional correction of the thoracic curve and sacral slope. ${ }^{7}$ A major goal of lumbar lordosis restoration is to achieve a degree of correction comparable to the patient's pelvic incidence.

Currently, 3 commonly used intraoperative techniques for restoring lumbar lordosis include cantilever bending, in situ bending, and compression and/or distraction of the screws along posterior fusion rods. ${ }^{11}$ These techniques might be used in combination with spine osteotomies, such as Ponte, Smith-Petersen, and pedicle subtraction osteotomy. All 3 corrective maneuvers require the surgeon to impart nontrivial forces to the posterior fusion instrumentation. The most common postoperative complications reported in the literature include proximal junctional failures, such as hyperkyphosis of the upper levels adjacent 
TABLE 1. Demographics and bone quality scores for the study population

\begin{tabular}{cccccrrrrr}
\hline & & & & & \multicolumn{4}{c}{ T-Score } \\
\cline { 7 - 10 } Specimen No. & Sex & Age (yrs) & Height (in) & Weight (lbs) & L-1 & L-2 & L-3 & L-4 \\
\hline 1 & Male & 71 & 71 & 150 & 0.2 & 0.8 & 1.5 & 0.3 \\
\hline 2 & Male & 54 & 66 & 180 & -1.9 & -1.5 & -1.5 & -1.6 \\
\hline 3 & Male & 70 & 72 & 240 & -0.6 & 0.0 & 1.2 & 1.7 \\
\hline 4 & Male & 75 & 74 & 140 & -2.3 & -0.1 & -1.5 & -1.2 \\
\hline 5 & Male & 57 & 70 & 160 & -1.8 & -2.2 & -2.8 & -2.5 \\
\hline
\end{tabular}

to the correction that are associated with failure of proximal pedicle screw fixation and/or vertebral compression fractures. ${ }^{4,9,10,13}$ Another common complication is failure to properly lordose the spine, as well as symptomatic rod fracture in the middle of the fusion constructs that is generally located at the apex of the lordotic curve in the rods. ${ }^{1}$ The occurrence of these complications is multifactorial and includes patient selection and construct design; however, we hypothesize that an important factor may be the intraoperative loads placed on the implants during these powerful correction maneuvers and the residual load states that may be left on the implants after the procedure.

To date, there have been no clinical studies that address the comparative performances of these 3 techniques. Besides the lack of definitive clinical data to support the use of one intraoperative technique over the others, no biomechanical evidence exists to guide surgeons in their choice of procedures for restoring lordosis. Prior studies have focused on implant loads in the surgical management of cervical $^{5,8}$ and lumbar ${ }^{14,15}$ degenerative diseases and adolescent idiopathic scoliosis. ${ }^{16}$ Studies that investigated the loads on posterior stabilization systems used a quasistatic loading approach during multiaxial bending, but did not address intraoperative loading. ${ }^{12,14,15}$ The loading conditions imparted on the spinal tissue and instrumentation during lumbar deformity correction remain unclear in the adult population.

There is a need for data on the biomechanical demands placed on spinal instrumentation during the restoration of lumbar lordosis in the intraoperative setting in order to elucidate if these demands may approach the threshold for intraoperative instrumentation failure. The goal of our study was to characterize the loads placed on instrumentation during 3 of the most common corrective maneuvers. Our interest was not to investigate the differences in loading between osteotomy techniques, but rather to investigate the differences in loading between correction techniques, regardless of osteotomy technique. We aimed to produce similar corrections on our group of whole-torso cadaver test specimens. We aimed to measure the peak forces in the pullout and insertion directions as well as the total range of forces experienced by the instrumentation during the correction. Finally, we aimed to measure the residual forces experienced by the instrumentation in the immediate postoperative period. We hypothesized that substantial differences in preoperative and residual loading would exist between the 3 corrective maneuvers and that the largest loads during surgery would be experienced at the ends of the construct (i.e., at L-1 and L-5).

\section{Methods}

\section{Specimen Inclusion and Exclusion Criteria}

Five noneviscerated, fresh-frozen cadaveric torsos (cervical to midfemur) were acquired for this study. All specimens were male to ensure high bone density. The demographics of each specimen are detailed in Table 1. Dual-energy x-ray absorptiometry scans were obtained prior to testing to omit any specimens with severe deformity and/or osteoporosis (mean T-score $\leq-2.5$ ). Specimens with moderate-to-severe deformity (e.g., scoliosis, kyphosis, degenerative disc disease with an L1-S1 Cobb angle $>10^{\circ}$ ) were removed from the study. A pretest manual evaluation was performed on all specimens, with none noted as being unusually stiff or flexible.

\section{Surgical Simulation of Corrective Maneuvers}

Each specimen underwent 3 lordosis restoration procedures in series, namely 1) cantilever bending, 2) in situ rod bending, and 3) compression/distraction. The test order was randomized among specimens. To characterize the extent of tissue fatigue from undergoing multiple procedures, the first procedure was repeated at the end of testing. Any differences in outcomes between the first and last procedure were reported. In total, 20 surgical simulations were conducted, with 4 procedures per specimen. All procedures were performed by a board-certified orthopedic surgeon and fellows specializing in spine surgery (D.K., C.T., and A.I.). A standard midline approach was taken to expose the posterior elements from L-1 to L-5. For cantilever bending, the rods were pre-bent to $60^{\circ}$ of lordosis (the upper limit of the normal range of lumbar lordosis), placed into the L-5 screw saddle, and locked in place with a set screw. The rod was sequentially reduced into the other screw saddles, climbing 1 screw at a time from L-4 to L-1 using a combination of manual pressure down on the free end of the rod along with the rod pusher and rocker-bottom reducer. The resulting L1-5 lordosis was measured on the fluoroscopic images using an electronic Cobb meter.

For in situ bending, the rod was minimally contoured and placed into the pedicle screw saddles. Once locked, the rod was contoured into lordosis using in situ sagittal benders. Sequential bending was performed on both sides until the maximal possible lordosis of $60^{\circ}$ was achieved or screw pullout was noted. In the event of screw pullout, the vertebrae were cemented. At the end of the maneuver, the final L1-5 lordosis was recorded.

For compression/distraction, the rod was contoured to the bendable rod template and placed into the pedicle screw 


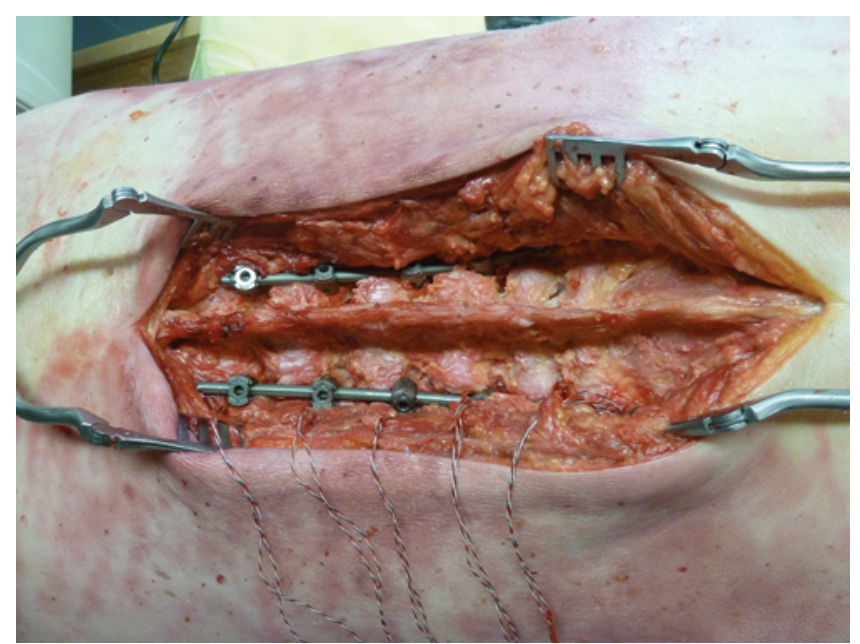

FIG. 1. Screw configuration with the posterior elements exposed and strain gauges deployed unilaterally. Figure is available in color online only.

saddles. Set screws were placed and kept loose with the exception of the L-5 screw, which was tightened. The compressor tool was used to compress the L-5 and L-4 screws together, and the L-4 set screw was tightened. Then, the L-3 screw was compressed to the L-4 screw, and the cycle was repeated until L-1 for incremental gains of lordosis.

\section{Measurement of Forces on the Screws}

Standard titanium screws and cobalt-chrome rods were used for this study $(6.5 \times 45-\mathrm{mm}$ titanium pedicle screws and 5.5-mm cobalt-chrome rods; DePuy Spine) and deployed according to manufacturer's guidelines. Each pedicle screw was instrumented with 2 uniaxial strain gauges by a third-party firm (Hitec Sensor Solutions, Inc.).
The strain gauges were adhered to a screw shaft below the tulip and sealed with a rubber resin to isolate them from wet-tissue exposure. The strain gauges were oriented to measure loads in the anteroposterior direction and placed $180^{\circ}$ apart about the circumference of the screw. The sensors were calibrated to measure the pullout force by developing a linear regression to establish the relationship between the standard $\mathrm{mV} / \mathrm{V}$ strain gauge output and the desired load output in Newtons. Instrumented screws were placed unilaterally prior to the first corrective procedure (Fig. 1). The screws remained implanted throughout the duration of the 4 procedures. For every trial, the first fusion rod was deployed on the instrumented side to observe the highest loading measurements possible. To allow for tissue relaxation, a minimum of 15 minutes was taken between procedures. Real-time measurements of the load were captured throughout the duration of each procedure with a data acquisition frequency of $200 \mathrm{msec}$. Data acquisition began after screw insertion and before rod deployment. At the completion of each bilateral procedure, screw-loading measurements continued for a minimum of 15 minutes to allow for relaxation of the soft tissue. From these data, 4 measurements were calculated. The methods used to calculate the intraoperative load parameters are displayed in Fig. 2. Two outcomes-peak intraoperative pullout and the push-in force at each screw-were calculated as the peak load in the pullout and insertion directions at any point during surgery. The total range of forces at each screw was calculated as the difference between the peak pullout and push-in forces during a single surgery. Residual pullout force at each screw was calculated as the amount of force in the pullout direction at 15 minutes after the completion of the surgery.

\section{Measurement of Lordosis Correction}

During each surgical simulation, the specimens were

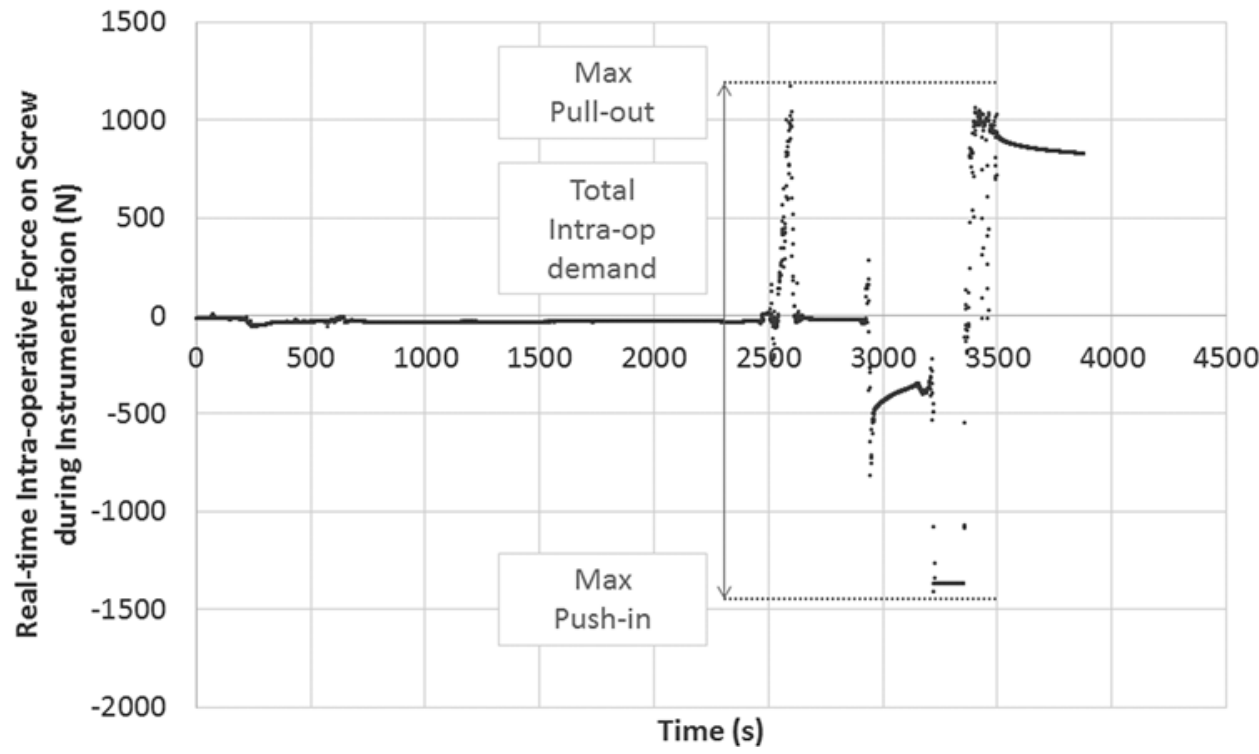

FIG. 2. Representative chart of real-time force measurements during a lordosis restoration procedure. The intraoperative outcome measures are labeled: max pullout, max push-in, and total demand. 
TABLE 2. Results of all outcome measures from testing each correction maneuver and vertebral level*

\begin{tabular}{|c|c|c|c|c|c|c|}
\hline Level & Procedure & Correction $\left({ }^{\circ}\right)$ & Peak Intraop Pullout (N) & Peak Intraop Push-In (N) & Total Intraop Demand (N) & Residual Force (N) \\
\hline $\mathrm{L}-1$ & Cantilever & $10.4 \pm 4.04$ & $555.08 \pm 229.07$ & $-226.40 \pm 152.72$ & $781.48 \pm 272.05$ & $138.30 \pm 99.16$ \\
\hline L-2 & Cantilever & & $947.16 \pm 321.01$ & $-880.56 \pm 749.26$ & $1827.73 \pm 1070.27$ & $569.54 \pm 391.03$ \\
\hline L-3 & Cantilever & & $521.25 \pm 211.11$ & $-973.10 \pm 1120.84$ & $1494.36 \pm 1329.97$ & $356.50 \pm 289.86$ \\
\hline$L-4$ & Cantilever & & $1281.32 \pm 619.57$ & $-1910.00 \pm 1641.71$ & $3191.28 \pm 1621.90$ & $1451.18 \pm 1064.38$ \\
\hline L-5 & Cantilever & & $220.10 \pm 94.68$ & $-814.35 \pm 85.65$ & $1034.46 \pm 9.03$ & $883.45 \pm 12.06$ \\
\hline L-1 & Comp/distraction & $12.2 \pm 5.63$ & $518.42 \pm 88.52$ & $-448.20 \pm 359.11$ & $966.67 \pm 333.07$ & $291.59 \pm 167.43$ \\
\hline $\mathrm{L}-2$ & Comp/distraction & & $867.58 \pm 345.35$ & $-532.69 \pm 439.07$ & $1400.27 \pm 784.42$ & $398.04 \pm 468.73$ \\
\hline L-3 & Comp/distraction & & $766.13 \pm 726.67$ & $-1895.20 \pm 1535.98$ & $2661.33 \pm 1525.24$ & $604.99 \pm 407.14$ \\
\hline L-4 & Comp/distraction & & $1896.25 \pm 970.69$ & $-1855.10 \pm 1169.37$ & $3751.37 \pm 2059.73$ & $696.07 \pm 440.11$ \\
\hline L-5 & Comp/distraction & & $805.61 \pm 197.99$ & $-197.96 \pm 180.07$ & $1003.57 \pm 378.06$ & $526.24 \pm 580.87$ \\
\hline L-1 & In situ & $10.0 \pm 5.05$ & $744.67 \pm 230.32$ & $-1134.70 \pm 933.08$ & $1879.32 \pm 1064.06$ & $398.68 \pm 245.52$ \\
\hline L-2 & In situ & & $687.42 \pm 130.76$ & $-637.25 \pm 171.89$ & $1324.67 \pm 41.13$ & $424.02 \pm 460.25$ \\
\hline L-3 & In situ & & $1297.83 \pm 831.09$ & $-1653.10 \pm 1278.27$ & $2950.92 \pm 2109.32$ & $545.64 \pm 422.35$ \\
\hline L-4 & In situ & & $2006.78 \pm 1087.87$ & $-2253.30 \pm 949.14$ & $4260.06 \pm 1888.71$ & $870.81 \pm 434.79$ \\
\hline$L-5$ & In situ & & $561.50 \pm 516.49$ & $-683.35 \pm 258.52$ & $1244.85 \pm 775.02$ & $616.87 \pm 389.47$ \\
\hline
\end{tabular}

Comp = compression.

* Values are presented as the mean \pm SD.

placed in the prone position on a C-arm-compatible table. Preliminary anteroposterior and lateral radiographs (Philips BV Pulsera) were taken preexposure to serve as the baseline for measuring lordotic correction. Fluoroscopic images were obtained as needed to assist in hardware deployment and ensure a final target lordotic correction of $10^{\circ}$. Anteroposterior and lateral images were obtained following the completion of each procedure in order to measure lordotic correction.

\section{Outcome Measures and Statistical Analysis}

The outcome measures were reported as follows: 1) degree of correction; 2) peak intraoperative pullout force at each level; 3) peak intraoperative push-in force at each level; 4) total intraoperative demand at each level; and 5) residual pullout force. The means and standard deviations for each outcome measure were calculated and compared for significant differences utilizing the Student t-test of repeated measures. An alpha value equal to 0.05 was taken as significant for all comparisons. All data are presented as mean \pm standard deviation.

\section{Results}

The average starting lordosis for the group of specimens was $28^{\circ} \pm 13^{\circ}$, indicating no gross deformities of the spine. The mean lordosis correction ranged from $10.0^{\circ}$ to $12.2^{\circ}$; however, no statistically significant differences $(p>0.05)$ were observed in the degree of correction achieved between any of the maneuvers (Table 2). The largest pullout forces on the screws during surgery were experienced at L-4 consistently across all maneuvers (Fig. 3). The smallest pullout forces were observed at the ends of the construct at the L-1 and L-5 levels. All maneuvers produced statistically similar peak pullout forces at each level. A similar trend was observed in the measurement of the peak push-in forces (Fig. 3). Analysis of the total intraoperative demand on the screws (difference between peak pullout and push-in) revealed statistically significantly lower loads at the L-1 and L-3 levels during the use of the cantilever maneuver (Fig. 4). The forces on the screws decreased during the immediate postoperative period (i.e., the residual force on screws; Fig. 5) compared with their corresponding peak pullout and push-in forces at each level and with each contour technique. On average, the forces decreased $53 \%$ during the immediate postoperative period. A significant difference in the residual force on L-1 was detected between the cantilever and in situ bending techniques (Fig. 5). The order of the test procedures did not affect outcomes; no differences in outcomes were observed between the first and fourth procedures.

\section{Discussion}

We observed substantial differences in the forces exerted on the pedicle screws during and after our 3 corrective maneuvers. The goal of this study was to characterize the demand on posterior fusion instrumentation for lordosis restoration procedures between 3 of the most common surgical techniques. We aimed to measure peak intraoperative and residual loads on the screws during the procedures that produced similar overall lordotic corrections. The in situ bending maneuver produced the largest total demand and residual loads on the L-1 screw, while cantilever bending produced the smallest. This may be due to the relative differences in the number of screws secured to the rod during each maneuver and the order in which the rods are contoured and the screws are secured. During in situ bending, all of the screws are secured to the rod before contouring. This allows the L-1 screw to experience loading during the contouring maneuvers at other levels. The L-1 screw is not secured to the rod during cantilever 


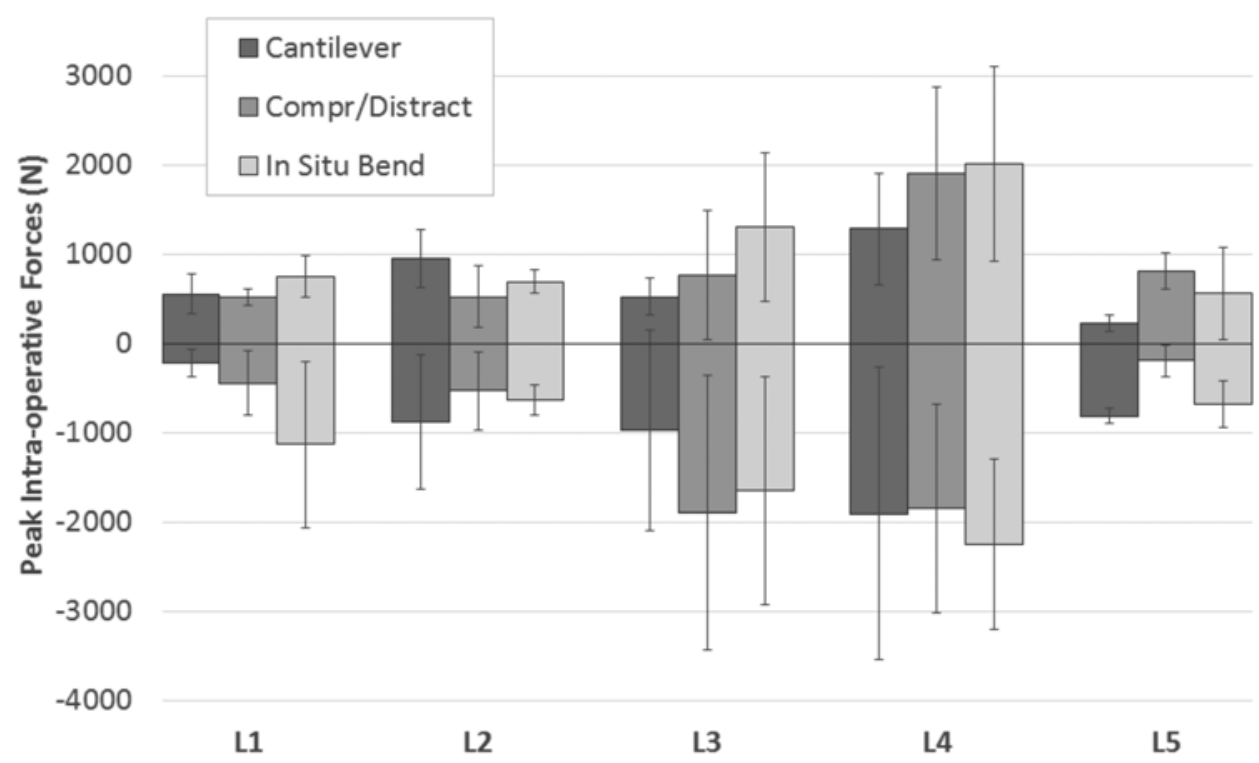

FIG. 3. Intraoperative forces observed at each level (L1-5). Values greater than 0 indicate the pullout force. Values less than 0 indicate the push-in force. Compr/distract = compression/distraction. All data are represented as the mean \pm standard deviation.

bending at other levels; therefore, the screw does not experience loads during those maneuvers. For example, the L-1 screw during in situ bending of L3-4 will experience some loads due to the distribution of the forces through the rod. The L-1 screw during cantilever bending at this level will not. This concept may have contributed to the significant differences in the total intraoperative demand we observed. The other difference we observed between maneuvers-difference in residual loads-may also be due to the L-1 screw being secured to the rod before the contour. The force required to contour the rod and lordose L1-2 is higher than the force required to only lordose
L1-2 to a precontoured rod. While a portion of the force imparted to the screw/rod construct in the in situ bending configuration will be borne by the rod, our evidence suggests a significant portion is imparted to the screw, and that portion exhibits substantial residual loads. We had hypothesized that the ends of the construct would experience the largest loads during corrective maneuvers. This was refuted by our data in every mode of measurement (intraoperative and residual loads). We observed the opposite finding, where the levels in the middle of the construct experienced substantially higher loads. This may be due to our strategy of prescribing the smallest radii of the

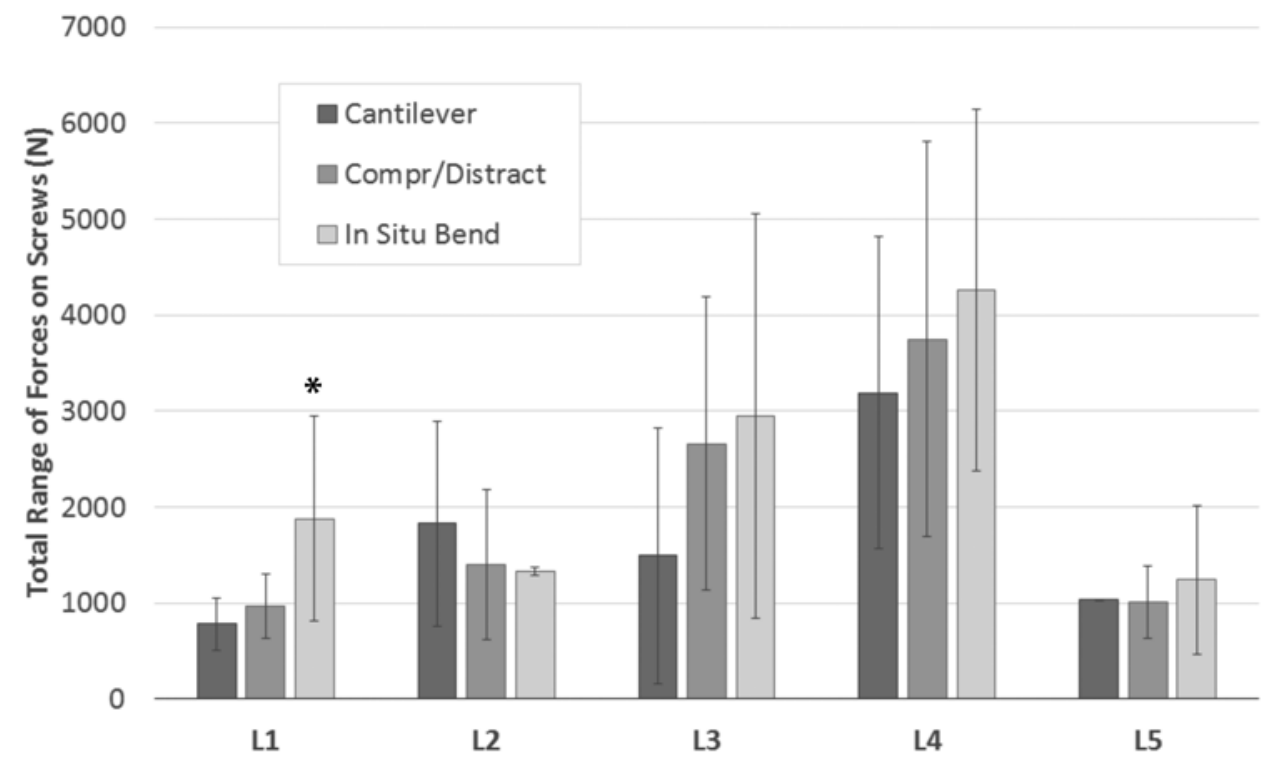

FIG. 4. Intraoperative demand observed at each level (L1-5). The asterisk indicates a statistically higher load than cantilever bending within the same level. All data are represented as the mean \pm standard deviation. 


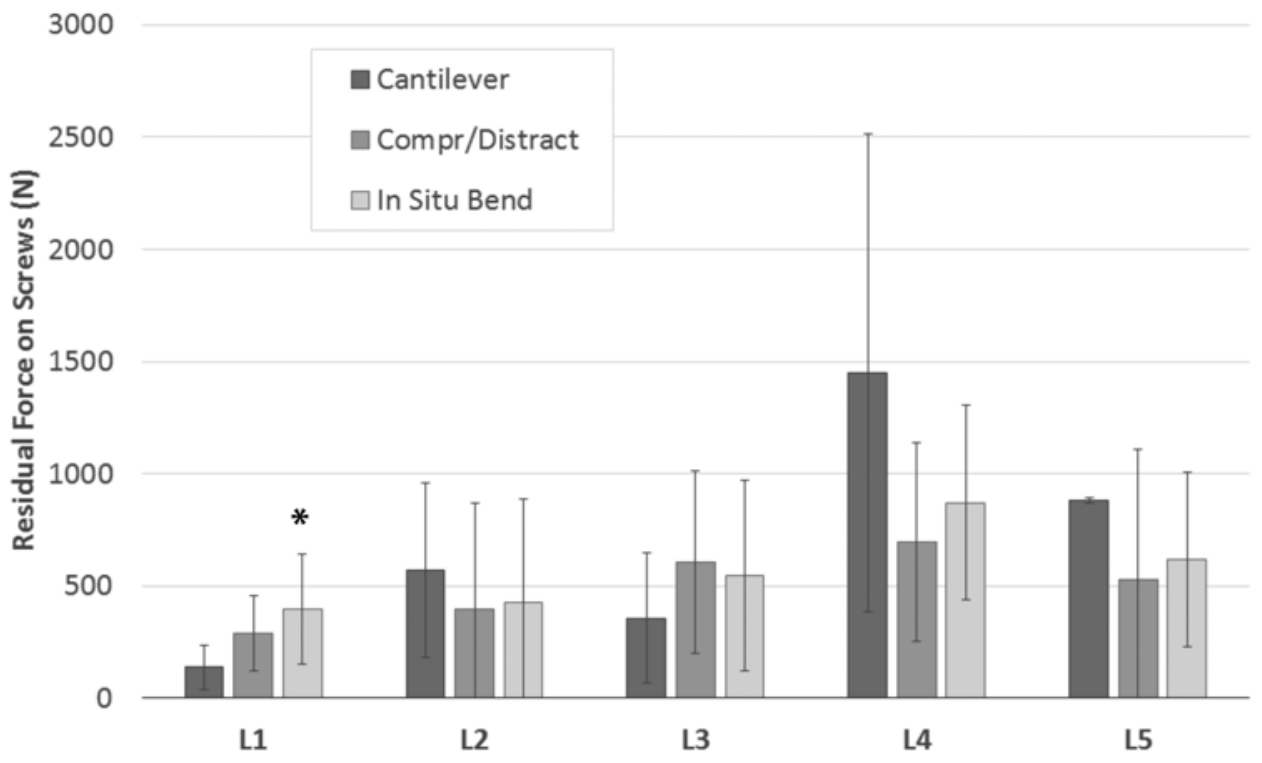

FIG. 5. Average residual load observed at each level (L1-5). The asterisk indicates statistically higher load than cantilever bending within the same level. All data are represented as the mean \pm standard deviation.

curvature to the middle of the construct. Smaller radii of curvature require larger loads to be imparted on the rod during in situ bending and larger loads to be imparted on the screws during cantilever bending. Interestingly, our data suggest that these differences in large intraoperative forces between levels do not subside during the immediate postoperative period. In fact, the difference in residual forces between 2 levels-for example, L-3 and L-4-may be larger than $1000 \mathrm{~N}$. This difference in forces indicates that a substantial shear load (larger than $1000 \mathrm{~N}$ ) on the rod may exist.

The degrees of correction for each procedure were found to be of the same order of the clinically observed corrections for lordosis restoration procedures. ${ }^{2,17}$ Previous studies analyzed the quasistatic loading profiles of posterior fusion instrumentation. The results of these studies are not directly comparable to the presented study, as they measure and report the loads in fusion rods rather than pedicle screws. ${ }^{12,14}$ Another study observed the loads on implanted pedicle screws via instrumented screws that were implanted in a lower-lumbar cadaveric model under range of motion testing. ${ }^{15}$ However, the results of this study measured torque rather than axial load and did not include the fusion of levels via posterior fusion rods. Other studies observed the intraoperative loads in cervical cases and reported the force required to distract screws via a calibrated Casper distractor, rather than the loads present in the posterior fusion instrumentation..$^{5,8}$ The intraoperative loads applied to the posterior fusion instrumentation in the cervical spine were on the order of $148.7 \pm 51.5 \mathrm{~N},{ }^{8}$ which is much lower than the loads typically observed in the lumbar spine. The tested single-level lumbar pedicle screw pullout strength has been reported to reach over $2000 \mathrm{~N} .{ }^{17}$ The maximum single-level pullout loads observed in this study averaged $2006.8 \mathrm{~N}$ (at the L-4 level during in situ bending). The average pullout strength observed in the previous study was over $1500 \mathrm{~N}$. The other 2 maneuvers evaluated in our study produced loads approaching and exceeding this reported average strength. These findings suggest that severe rod contouring places the pedicle screws at risk for failure, thereby weakening the fusion construct.

The strengths of our study include the use of a full cadaver model, thereby simulating the operative environment for instrumentation. The use of a full torso also provided an accurate contribution of the soft tissues to the resistance of the sagittal plane correction, especially during our measurement of the postoperative loads. All procedures were performed by the same surgeons, ensuring consistency in technique throughout the study. To the best of our knowledge, this is the first report to compare forces between 3 different techniques for correcting sagittal plane deformity in the lumbar spine.

This study has limitations. There was little control of the individual spine specimen's resistance to correction. No osteotomies were performed and no measurements of facet size or arthropathy were collected, which may have significant effects on the specimen's resistance to correction; however, no gross deformities were noted by the fellowship-trained spine surgeon, and the starting lordosis for each specimen was within the range for normal adults. Another limitation was the measurement of forces on only 1 side of the bilateral implant construct. We did not observe loading on the contralateral screws; however, the forces were measured on the side that was first contoured and secured to the rod. Another limitation is the small sample size. Several results in this study demonstrated a trend but lacked statistical significance, which may be due to the small number of cadavers used (Type II error). The cadaveric model did not account for the postoperative effects of ambulation, axial loading from being upright, or fatigue on instrumentation over time. Another limitation is the use of instrumentation that extended to only L-1 in this study. The effect of a longer construct that includes the thoracic spine on instrumentation was not studied. Proxi- 
mal junctional kyphosis was also not accounted for in this biomechanical study. Future work may study the effects of performing osteotomy to optimize correction. Partially destabilizing the spine with a 1- or 3-column osteotomy (e.g., Smith-Petersen or pedicle subtraction osteotomy) may be instrumental to achieving the desired correction without imparting excessive loads onto the instrumentation; however, our choice to correct without osteotomy was made to generalize our results as much as possible.

\section{Conclusions}

The results of this study indicate that in situ bending demands the highest mechanical performance from posterior fusion instrumentation in comparison with both cantilever bending and compression/distraction techniques. The results indicate a greater chance of intraoperative instrumentation failure and postoperative proximal pedicle screw pullout with in situ bending. The results of this study are aimed to optimize the correction strategies for lumbar lordosis restoration and indicate that further studies with larger sample sizes are warranted to elucidate the potential risks for instrumentation failure.

\section{Acknowledgments}

Financial support for this study was provided by the AO Foundation.

\section{References}

1. Aryan HE, Newman CB, Lu DC, Hu SS, Tay BK, Bradford DS, et al: Relaxation of forces needed to distract cervical vertebrae after discectomy: a biomechanical study. J Spinal Disord Tech 22:100-104, 2009

2. Belmont PJ Jr, Polly DW Jr, Cunningham BW, Klemme WR: The effects of hook pattern and kyphotic angulation on mechanical strength and apical rod strain in a long-segment posterior construct using a synthetic model. Spine (Phila Pa 1976) 26:627-635, 2001

3. Cho KJ, Suk SI, Park SR, Kim JH, Kang SB, Kim HS, et al: Risk factors of sagittal decompensation after long posterior instrumentation and fusion for degenerative lumbar scoliosis. Spine (Phila Pa 1976) 35:1595-1601, 2010

4. Cho KJ, Suk SI, Park SR, Kim JH, Kim SS, Choi WK, et al: Complications in posterior fusion and instrumentation for degenerative lumbar scoliosis. Spine (Phila Pa 1976) 32:2232-2237, 2007

5. Francke EI, Demetropoulos CK, Agabegi SS, Truumees E, Herkowitz HN: Distractive force relative to initial graft compression in an in vivo anterior cervical discectomy and fusion model. Spine (Phila Pa 1976) 35:526-530, 2010

6. Glassman SD, Bridwell K, Dimar JR, Horton W, Berven S, Schwab F: The impact of positive sagittal balance in adult spinal deformity. Spine (Phila Pa 1976) 30:2024-2029, 2005

7. Jang JS, Lee SH, Min JH, Maeng DH: Changes in sagittal alignment after restoration of lower lumbar lordosis in patients with degenerative flat back syndrome. J Neurosurg Spine 7:387-392, 2007

8. Kettler A, Niemeyer T, Issler L, Merk U, Mahalingam M, Werner K, et al: In vitro fixator rod loading after transfo- raminal compared to anterior lumbar interbody fusion. Clin Biomech (Bristol, Avon) 21:435-442, 2006

9. Kim YJ, Bridwell KH, Lenke LG, Rhim S, Kim YW: Is the T9, T11, or L1 the more reliable proximal level after adult lumbar or lumbosacral instrumented fusion to L5 or S1? Spine (Phil Pa 1976) 32:2653-2661, 2007

10. Maruo K, Ha Y, Inoue S, Samuel S, Okada E, Hu SS, et al: Predictive factors for proximal junctional kyphosis in long fusions to the sacrum in adult spinal deformity. Spine (Phila Pa 1976) 38:E1469-E1476, 2013

11. Mermer MJ, Boachie-Adjei O, Rawlins BA, Peskin M, Bhatnagar R: Comprehensive analysis of cantilever, translational, and modular corrective techniques in adults with scoliosis treated with surgery to the sacropelvis. J Spinal Disord Tech 19:513-522, 2006

12. Meyers K, Tauber M, Sudin Y, Fleischer S, Arnin U, Girardi F, et al: Use of instrumented pedicle screws to evaluate load sharing in posterior dynamic stabilization systems. Spine J 8:926-932, 2008

13. O'Leary PT, Bridwell KH, Lenke LG, Good CR, Pichelmann MA, Buchowski JM, et al: Risk factors and outcomes for catastrophic failures at the top of long pedicle screw constructs: a matched cohort analysis performed at a single center. Spine (Phila Pa 1976) 34:2134-2139, 2009

14. Scheer JK, Tang JA, Buckley JM, Deviren V, Pekmezci M, McClellan RT, et al: Biomechanical analysis of osteotomy type and rod diameter for treatment of cervicothoracic kyphosis. Spine (Phila Pa 1976) 36:E519-E523, 2011

15. Scheer JK, Tang JA, Deviren V, Acosta F, Buckley JM, Pekmezci M, et al: Biomechanical analysis of cervicothoracic junction osteotomy in cadaveric model of ankylosing spondylitis: effect of rod material and diameter. J Neurosurg Spine 14:330-335, 2011

16. Scheer JK, Tang JA, Deviren V, Buckley JM, Pekmezci M, McClellan RT, et al: Biomechanical analysis of revision strategies for rod fracture in pedicle subtraction osteotomy. Neurosurgery 69:164-172, 2011

17. Wedemeyer M, Parent S, Mahar A, Odell T, Swimmer T, Newton P: Titanium versus stainless steel for anterior spinal fusions: an analysis of rod stress as a predictor of rod breakage during physiologic loading in a bovine model. Spine (Phila Pa 1976) 32:42-48, 2007

\section{Disclosures}

Dr. Ames is an employee of UCSF, works as a consultant for DePuy, Medtronic, and Stryker, holds patents with Fish \& Richardson PC, and owns stock in Doctors Research Group and Visualase. Dr. Telles works as a consultant for Spineart USA and Misonix.

\section{Author Contributions}

Conception and design: Leasure, Kuo, Martin. Acquisition of data: Kuo, Martin. Analysis and interpretation of data: all authors. Drafting the article: Leasure, Kuo, Telles. Critically revising the article: Leasure, Kuo, Telles, Iezza, Ames, Kondrashov. Reviewed submitted version of manuscript: Leasure, Kuo, Telles, Iezza, Ames, Kondrashov. Approved the final version of the manuscript on behalf of all authors: Leasure. Administrative/technical/material support: Leasure, Telles, Iezza, Ames, Kondrashov. Study supervision: Telles, Ames, Kondrashov.

\section{Correspondence}

Jeremi Leasure, The Taylor Collaboration, 450 Stanyan St., San Francisco, CA 94117. email: jleasure@taylorcollaboration.org. 